\title{
A REVIEW: FILM FORMING GEL NOVEL DRUG DELIVERY SYSTEM
}

\author{
SWAPNIL S. BORNARE ${ }^{*}$, SMITA S. AHER ${ }^{2}$, RAVINDRANATH B. SAUDAGAR ${ }^{3}$
}

${ }^{*}$ Department of Quality Assurance Techniques, R. G. Sapkal College of Pharmacy, Anjaneri, Nashik 422213, Maharashtra, India

Email: swapbornare8@gmail.com

Received: 24 Dec 2017, Revised and Accepted: 05 Feb 2018

\begin{abstract}
Film forming gels are a novel approach in this area that might present an alternative to the conventional dosage forms used on the skin, such as ointments, creams, gels or patches. The polymeric solution is applied to the skin as a liquid and forms an almost invisible film in situ by solvent evaporation. Transdermal drug delivery system (TDDS) and dermal drug delivery system can provide some desirable performances over conventional pharmaceutical dosage formulations, such as avoiding gut and hepatic first-pass metabolism, improving drug bioavailability, reducing dose frequency and stabilizing drug delivery profiles. The aim of this review was to search for alternatives to the conventional forms in order to reduce skin irritation, improve skin adhesion properties, enhance the drug release and increase the patient acceptability from an aesthetic perspective. Because of their peculiar rheological behaviour, polymeric gels are beneficial in terms of ease of preparation, ease of application, adhesion to the application surface and ability to deliver a wide variety of drugs.
\end{abstract}

Keywords: Film forming gels, Transdermal drug delivery, Gelling agents

(C) 2018 The Authors. Published by Innovare Academic Sciences Pvt Ltd. This is an open access article under the CC BY license (http://creativecommons.org/licenses/by/4.0/) DOI: http://dx.doi.org/10.22159/ijcpr.2018v10i2.25886

\section{INTRODUCTION}

The skin is the most readily accessible organ of the body and acts as a barrier against the micro and macromolecules of the environment because of its low permeability to such substances [1]. The skin of an average adult body has approximately $2 \mathrm{~m} 2$ surface area and it receives about one-third of the total blood circulating throughout the body [2]. Percutaneous absorption of drug through skin mainly occurs via stratum corneum. Stratum corneumis made up of dead, keratinized epidermal cells having thickness of $10 \_\mathrm{m}$ and acts as a barrier for permeation of drugs. Therefore transport of drug molecules across the skin is difficult [3].

The goal of drug administration through skin is for topical treatment of skin diseases or for transdermal absorption of drugs in the systemic circulation. The topical route offers a large and varied surface in addition to the ease of application via self-administration and provides an alternative to oral delivery of drugs as well as hypodermic injection [4]. The rate and extent of drug absorption through skin depends on the skin physiology and physicochemical properties of drugs as well as the delivery system. The current dosage forms, i.e. patches, ointments, creams, etc., are associated with several limitations. Patches have various disadvantages, most commonly skin irritation [5], because of their occlusive properties causing obstruction of sweat ducts, which in turn prevents loss of water vapor from skin surface, difficulty in applying on the curved surfaces, pain while peeling off and poor aesthetic appeal. Semisolid preparations like creams and ointments overcome some of these drawbacks but have other limitations. These do not ensure persistent contact with the skin surface and can be easily wiped off by patient's clothes [6]. Hence repeated application is required in case of chronic diseases like athlete's foot, ringworm and candidiasis [7]. Also these leave a sticky and greasy feel after application leading to poor patient compliance $[8,9]$. Therefore there is a need for development of a dosage form which permits less frequent dosing by maintaining a close contact with the skin for prolonged time period thereby improving the patient compliance. Film forming system (FFS) is a novel approach which can be used as an alternative to conventional topical and transdermal formulations. It is defined as non-solid dosage form that produces a film in situ, i.e. after application on the skin or any other body surface. These systems contain the drug and film forming excipients in a vehicle which, upon contact with the skin, leaves behind a film of excipients along with the drug upon solvent evaporation. The formed film can either be a solid polymeric material that acts as matrix for sustained release of drug to the skin or a residual liquid film which is rapidly absorbed in the stratum corneum [10].

\section{Mechanism of film formation and permeation}

Film forming system is applied directly to the skin and it forms a thin, transparent film in situ upon solvent evaporation as shown in fig. 1. After application of the formulation to the skin, the composition of the film forming system changes significantly due to the loss of the volatile components of the vehicle which results in formation of residual film on the skin surface. In this process the concentration of drug increases, reaching saturation level and with the possibility of reaching super saturation level on the skin surface. Supersaturation results in the enhanced drug flux through the skin by increasing the thermodynamic activity of the formulation without affecting the skin's barrier, thereby reducing the side effects or irritation $[10,11]$.

The concept of supersaturation can be explained by the modified form of Fick's law of diffusion. Fick's law of diffusion given by Eq. (2.1):

$$
\mathrm{J}=\frac{\mathrm{DKCv}}{\mathrm{h}}
$$

Where

$J=$ rate of drug permeation per unit area of skin per unit time (flux)

$D=$ diffusion coefficient of drug

$C V=$ concentration of drug

$h=$ thickness of barrier to diffusion

From this equation, it is clear that the rate of drug permeation across the skin is proportional to the concentration of the drug. However this is true when the entire drug is dissolved in the vehicle. Equation (2.2) describes the modified form of Fick's law of diffusion:

$$
\mathrm{J}=\alpha \mathrm{D} / \gamma \mathrm{h}
$$

Where

a=thermodynamic activity of drug within formulation $\gamma=$ thermodynamic activity of drug within membrane

According to this equation, the flux of the drug is directly proportional to the thermodynamic activity of the system, which is related to saturation. However increasing the super saturation increases thermodynamic instability [12]. 


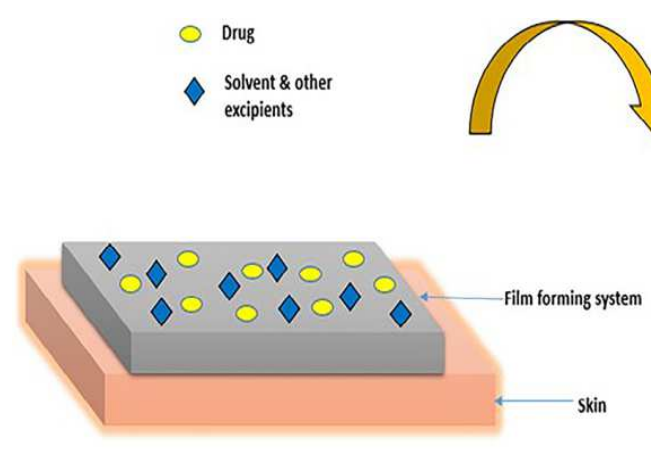

Before solvent evaporation

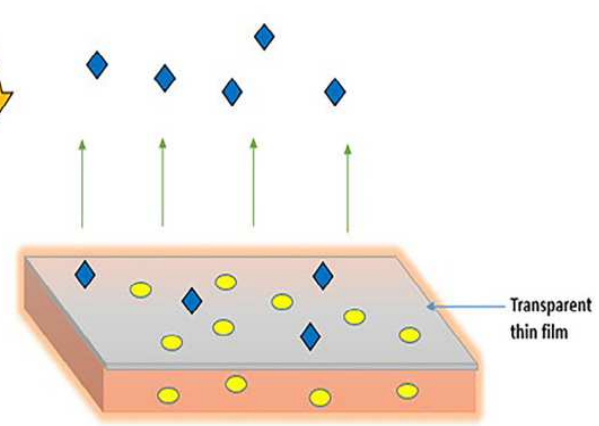

After solvent evaporation

Fig. 1: Mechanism of film formation

FFS creates supersaturated systems immediately after application to the skin, overcoming the problem of instability. Thus it improves the drug permeation through skin compared to other transdermal dosage forms. The delivery efficiency of the film forming solutions for ethinyl estradiol was investigated. The permeation of ethinyl estradiol from the film forming solution prepared with enhancer or without enhancer was compared to the permeation from the commercially available patch (EVRA®) through human epidermis in vitro. The film forming formulations showed a higher permeation than the commercial patch. Without enhancer the formulation transported more than double the ethinyl estradiol than the marketed patch. With enhancer, the formulation delivered about seven times as much ethinyl estradiol as that of the marketed patch. Thus these systems prove to be useful in enhancing the drug permeation.

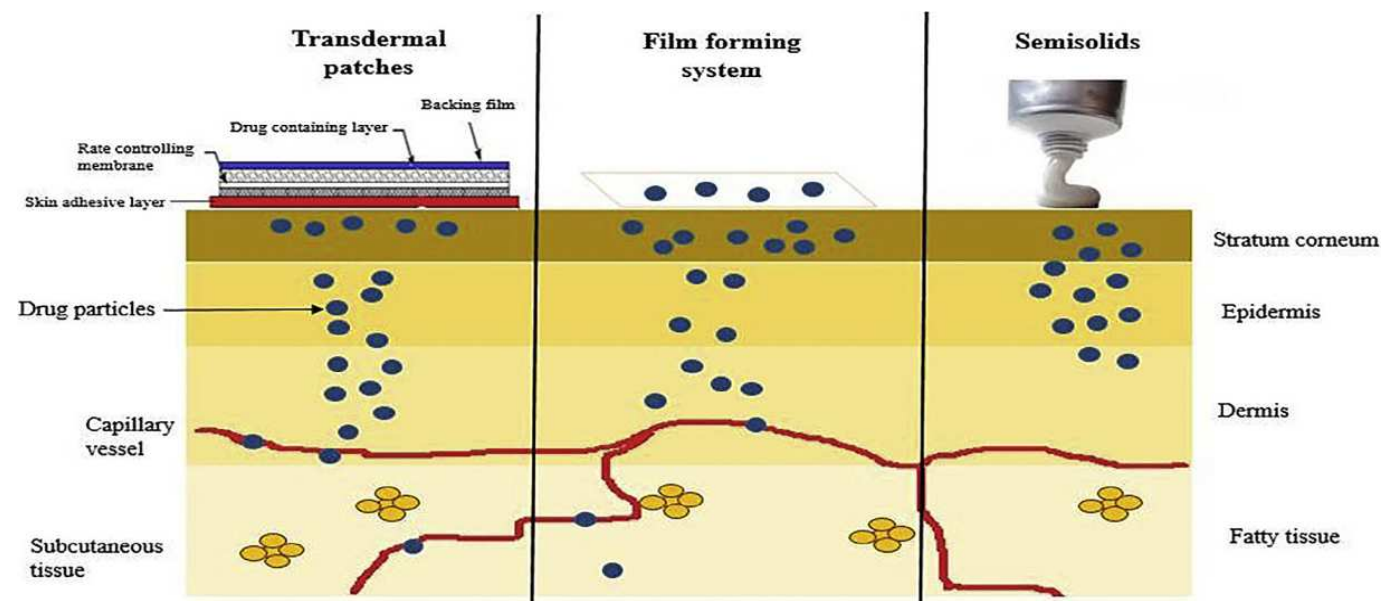

Fig. 2: Release Profile of the topical and transdermal drug delivery systems

\section{Considerations for the composition of film forming polymeric gels}

\section{Suitable drugs}

The usage of the developed formulations for dermatological indications is theoretically possible as well but cannot be recommended sometimes due to the nature of the solvent in the compositions. Dermatological diseases are often associated with inflamed skin where the administration of ethanolic solutions might be painful for the patient and thus not acceptable. For a transdermal application suitable drugs have to fulfil certain requirements that are independent of the dosage form [13]. Due to the fact that the skin is a very efficient protective barrier for the body, not merely against physical or microbiological noxes but also against drugs, only potent drugs are eligible for this application route with a daily dose of less than $10 \mathrm{mg}$. The size of the molecule is required to be small in order to provide a sufficient mobility in the skin structures (molecular weight below $500 \mathrm{Da}$ ). As the drug has to pass lipophilic as well as hydrophilic areas in the skin on its way into the systemic circulation it is advantageous if the drug is neither very hydrophilic nor extremely lipophilic ( $\log \mathrm{P}$ between 1 and 3). Therefore molecules with a pH value between 5 and 9 in aqueous solution are preferred for the transdermal application [17].
Further parameters that are beneficial for the transdermal delivery of a drug are a small number of hydrogen bonding groups $(<2)$ and a low melting point(less than $200^{\circ} \mathrm{C}$ ). The reservoir size of the dosage form is comparatively small due to the extreme thinness of the films (approximately 5-25 $\mu \mathrm{m}$ ). With a formulation dose of $10 \mathrm{mg} / \mathrm{cm} 2$ and an application area with an acceptable size of for instance 30 $\mathrm{cm} 2$ the total applied formulation dose would be $300 \mathrm{mg}$. In order to reach the limit daily dose that is assumed for transdermal patches (10 $\mathrm{mg} /$ day) more than $30 \%$ of the applied dose would have to be absorbed. Such a high absorption was not achieved with the steroidal hormones that were investigated in this work. With these drugs the absorption was clearly below $10 \%$ of the applied dose. Taking this into account it seems obvious that the film forming solutions will be mainly attractive for drugs that have.

- A high potency (example: the progestin Nestorone)

- A high skin permeability (example: nicotine)

- A high solubility in the solvent (example: ethinyl estradiol).

A high potency is beneficial as it results in low required daily doses for the drug. High skin permeability promotes a high exploitation of the thin reservoir provided a suitable polymeric matrix is given. A 
high solubility in the formulation, finally, allows high drug loadings and the establishment of a high gradient between formulation and skin. An efficient delivery system, meaning a high exploitation of the drug reservoir throughout the wearing time, is generally desirable for all drugs. If this cannot be achieved the film forming system might not be attractive for expensive drugs due to the considerable portion of drug that is wasted [15]. It remains subject to further research if the film forming solutions are suitable for drugs with a narrow therapeutic window as the kinetics of the delivery system are not yet known. With respect to the novelty of the film forming systems a thorough and individual evaluation for each new drug candidate is still inevitable until a clearer picture of the capabilities of the film forming solutions in general has been gained.

\section{Appropriate excipients}

\section{Polymer}

The polymer is required to form films at the skin surface temperature $\left(28{ }^{\circ} \mathrm{C}-32{ }^{\circ} \mathrm{C}\right)$ and should have a certain inherent flexibility and affinity to the skin to avoid the usage of excessive amounts of plasticizer. It has to be soluble in a highly volatile, skin friendly solvent. Moreover, strong gelling agents should be avoided as film former as they prevent an application of the formulation by spraying. In spite of the many requirements the polymer screening experiments have demonstrated that the majority of the tested polymers could be formulated into a film forming composition with suitable macroscopic properties. Only four of the 14 tested polymers (chitosan, poly-iso-butylene, polyvinyl alcohol, polyvinylpyrrolidone) lacked some of the required properties and were therefore abandoned. The fact that the successfully utilized polymers differed widely in their chemical structure indicates that the formulation of this dosage form is not limited to certain polymer groups. It is highly probable that many more candidates for film forming solutions can be identified among the numerous polymers that are available on the market. Although various polymers yielded films with suitable macroscopic properties, however, the permeation studies have shown that some polymers are superior to others with respect to the drug delivery. The results indicated that the polymers do not only immobilize the drugs in a matrix on the skin, but that they may also have an enhancing (in case of the DynamX® formulation) or a retarding (in case of the Eudragit RL formulation) effect on the drug permeation. These effects can result on the one hand from complex interactions of the polymeric formulation with the skin, on the other hand also from interactions of the polymer with the drug. The extent of the latter is specific for each drug-polymer combination, depending on the physicochemical properties of the two compounds such as charge or lipophilicity. This should be kept in mind for the selection of a film forming formulation for a new drug candidate [14].

\section{Solvent}

The solvent is also a very important compound in the film forming solution although it is not part of the actual film on the skin due to its quick evaporation. The solvent must offer sufficient solubility for the polymer as well as for the drug. Only a high solubilizing power of the solvent for the drug allows substantial variations of the drug loading to modulate the drug delivery to the skin. The solvent can also exert a direct influence on the drug flux. Depending on the nature of the solvent and its permeation enhancing properties it can promote the drug transport to different extents in spite of its short contact time with the skin. This should be kept in mind for a further formulation development. In addition to its solubilizing properties for the polymer and the drug a suitable solvent for a film forming solution is required to be highly volatile to provide short drying times and thus a good patient compliance. Together with the polymer it is supposed to spread well on the skin after application to produce a smooth film with a uniform thickness on the application site. Both requirements are not met for example by the solvent water. During the formulation experiments an aqueous chitosan formulation displayed unacceptably long drying times and an uneven spreading on the skin due to the high surface tension of the aqueous polymeric formulation. Consequently, water cannot be considered a suitable solvent for the formulation of a film forming polymeric composition. Solvents such as ethanol, isopropanol or ethyl acetate with a higher volatility and a better spreading are to be preferred [18].

\section{Plasticizer}

In polymerisc applications the main purpose of a plasticizer is to facilitate the film forming and to increase the flexibility of the resulting film. Additionally, the formulation experiments have shown that the skin adhesion of the films can be modulated with the help of plasticizers. The plasticizer has to be thoroughly selected with regard to the film former. It has to be miscible with the polymer to produce clear films with low visibility on the skin. Since the efficiency of a plasticizer is polymer dependant no general rule can be applied as to which plasticizer concentration is required to produce films with the desired properties. The individual determination of the adequate plasticizer content is inevitable. An insufficient amount of the excipient leads to brittle films with low skin adhesion. An excessive amount of plasticizer on the other hand results in smooth, but sticky films. Both situations are unacceptable for a reliable drug delivery by the film forming system and a good patient compliance. The plasticizer should preferably have a low skin permeability to prevent leaking from the formed film. A substantial leaking would not only raise safety concerns but would also lead to a deterioration of the film properties. In case of a loss of plasticizer the film becomes brittle and loses part of its adhesive properties. The acrylate polymers poly (ethyl acrylate-co-methyl methacrylate) Eudragit NE 40D as well as the silicone gum formed adequate films without the help of a plasticizing agent [16].

\section{Further excipients}

Apart from the basic compounds of a film forming polymeric solution (polymer, solvent and plasticizer) it can be appropriate to incorporate further excipients into the preparation. For some polymers such as the acrylate Eudragit E 100, it is beneficial to add a crosslinker (succinic acid) to the composition to improve the film stability. For some drugs, a solubilizer or co-solvent can be required in order to increase the drug loading of the formulation and the drug flux. Further examples for supplementary excipients are antioxidants to stabilize oxidation sensitive drugs in the preparation during storage, sun screens for the protection of photosensitive drugs or dyes to facilitate the localisation of the formed film for the patient. A precondition for the incorporation of further excipients is the compatibility of the materials with all other compounds. Furthermore it has to be kept in mind that every change in the film composition might negatively affect the macroscopic properties of the formed film such as stability, adhesion to the skin or stickiness of the outer surface of the film. Therefore it is advisable to re-evaluate the macroscopic properties of the formed film after any adjustment of the composition.

\section{Evaluation tests for film forming gels}

\section{Phase transition time}

Time needed by the gel to get converted into film is the phase transition time. One gram of gel was placed on a petri dish which was spread uniformly on it and kept on a hot plate at $37^{\circ} \mathrm{C}$ and time needed until gel converts into film was measured.

\section{Film weight}

One gram of the gel was placed on a petridish which was left for drying. After drying the resultant film was weighed on an electronic balance.

\section{Film thickness}

Film thickness was measured by verniercalipers/screw gauge. The gel was spread on an area of $5 \mathrm{~cm}^{2}$ demarcated on a petridish. This petridish was left overnight for drying and then the film was peeled off and the thickness was determined from three different points on the film.

\section{Rheological studies}

The Brookfield Viscometer LVDV II was used to determine the rheology of studied gels. Gels were placed under the viscometer using S 64 spindle to determine their viscosity. The viscosity was determined at different RPM of 10, 20, 50, 100 and the corresponding viscosity and torque were noted. 


\section{Spreadibility studies}

Minimum quantity of the formulation was placed between two glass plate and the glass plate on the top was gently slided on the bottom glass slide to determine the spreadibility of the formulation was measured on the basis of drag and slip characteristics of gels. A ground glass slide was fixed on this block. An excess of gel (about $2 \mathrm{gm}$ ) under study was placed on this ground slide. The gel was then sandwiched between this slide and another glass slide having the dimension of fixed ground slide and provided with the hook. A $1 \mathrm{Kg}$ weight was placed on the top of the two slides for $5 \mathrm{~min}^{*}$ to expel air and to provide a uniform film of the gel between the slides. Excess of the gel was scrapped off from the edges. The top plate was then subjected to pull of 80 gms. With the help of string attached to the hook and the time (in seconds) required by the top slide to cover a distance of 7.5 $\mathrm{cm}$ be noted. A shorter interval indicates better Spreadability. Spreadibility was calculated using the following formula:

$$
\text { Spreadibility }=\mathrm{M} \times \mathrm{L} / \mathrm{T}
$$

Where, $\mathrm{S}=$ Spreadibility, $\mathrm{M}=$ Weight in the pan (tied to the upper slide), $\mathrm{L}=$ Length moved by the glass slide and $\mathrm{T}=$ Time (in sec.) taken to separate the slide completely each other.

\section{CONCLUSION}

Film forming gels proves to be effective dosage form for the transdermal delivery of drugs. Also it remains adhered to the effected part for a longer period without getting rubbed off. It provides sustained effect and better relief than the conventional gels and frequent reapplication is not required. The concept of film forming gels can change to treatment concept of various diseases such as arthritis. A lot of work can be carried out in this field.

\section{AUTHORS CONTRIBUTIONS}

All the author have contributed equally

\section{CONFLICT OF INTERESTS}

Declared none

\section{REFERENCES}

1. Renata CV, Tatiele K, Silvia GS. Drug transport across skin. In: Muro S. editor. Drug Delivery across Physiological Barriers. Taylor and Francis Group LLC; 2016. p. 132-4.

2. Sharma N, Agarwal G, Rana A. A review: transdermal drug delivery system a tool for novel drug delivery system. Int J Drug Dev Res 2011;3:70-84.
3. Michaels AS, Chandrasekaran SK, Shaw JE. Drug permeation through human skin: theory and in vitro experimental measurement. AIChE J 1975;21:985-96.

4. Prausnitz MR, Langer R. Transdermal drug delivery. Nat Biotechnol 2008;26:1261-8.

5. Dhiman S, Singh GT, Rehni AK. Transdermal patches: a recent approach to new drug delivery system. Int J Pharm Pharm Sci 2011;3:26-34.

6. Advanced Film-Forming Agent for Transdermal Drug Delivery. Available from: http://marketplace.yet2.com/app/insight/ techofweek/28842?Sid=350. [Last accessed on 12 Dec 2016]

7. Fungal Infections of the Skin. Available from: http://www.webmd.com/skin-problems-andtreatments/guide/fungal-infections-skin\#1. [Last accessed on 24 Dec 2016]

8. Devaux S, Castela A, Archier E. Adherence to topical treatment in psoriasis: a systematic literature review. I Eur Acad Dermatol Venereol 2012;26:61-7.

9. Tan X, Feldman SR, Chang J. Topical drug delivery systems in dermatology: a review of patient adherence issues. Expert Opin Drug Delivery 2012;9:1263-71.

10. Mcauley WJ, Caserta F, Hoboken NJ. Film-forming and heated systems. In: Donnelly RF, Singh TRR. editors. Novel delivery systems for transdermal and intradermal drug delivery. United States: John Wiley and Sons; 2015. p. 97-107.

11. Frederiksen K, Guy RH, Petersson K. The potential of polymeric film-forming systems as sustained delivery platforms for topical drugs. Expert Opin Drug Delivery 2015; 13:349-60.

12. Brown MB, Jones SA, Limited M. Topical film-forming monophasic formulations. Patent CA2622624C; 2006.

13. Zurdo Schroeder I. Film forming polymeric solutions as drugdelivery systems for the skin, Saarland University, Saarbrücken, Germany; 2007. [Last accessed on 21 Dec 2016].

14. Naik A, Kalia YN, Guy RH. Transdermal drug delivery: overcoming the skin's barrier function. Pharm Sci Technol 2000;3:318-26.

15. Thomas BJ, Finnin BC. The transdermal revolution. Drug Discovery Today 2004;16:697-703.

16. Zhai H, Maibach HI. Occlusion vs. skin barrier function. Skin Res Technol 2002;8:1-6.

17. Alberti I, Grenier A, Kraus, Raus H, Carrara DN. Pharmaceutical development and clinical effectiveness of a novel gel technology for transdermal drug delivery. Exp Opin Drug Delivery 2005;2:935-50.

18. Lee JW, Park JH, Robinson JR. Bioadhesive-based dosage forms: the next generation. J Pharmaceutical Sci 2000;89:850-66. 